\title{
Cauda Equina Syndrome in Neurosarcoidosis
}

\author{
Karan Topiwala ${ }^{1}$, Subhendu Rath ${ }^{2}$, Annie Daniel ${ }^{1}$, Avinash Prasad ${ }^{3}$ \\ 1. Neurology, Hartford Hospital, Hartford, USA 2. Neurology, University of Michigan School of Medicine, Ann Arbor, \\ USA 3. Neurology, University of Connecticut School of Medicine, Hartford Hospital, Hartford, USA
}

Corresponding author: Karan Topiwala, topiwala.karan@gmail.com

\begin{abstract}
Neurosarcoidosis (NS) is a mimicker of many infectious, neoplastic, and inflammatory diseases. It most commonly involves the cranial nerves followed by meninges, ventricles, hypothalamic-pituitary axis, spinal cord, and brainstem/cerebellum. While NS myelopathy has been increasingly recognized, pathophysiological/prognostic and management principles in NS-mediated cauda equina (CE) and conus medullaris (CM) syndromes, which constitute a small and rare minority of this subset, remain elusive. We present the case of a 49 -year-old Hispanic man who developed a peripheral facial palsy and primary hypogonadism within a span of 12 months and eventually got diagnosed with NS after he presented with CE syndrome. We also performed an extensive literature review, with a discussion on the underlying pathophysiology and current management recommendations for NS-mediated CE/CM syndrome. CE/CM syndromes in a middle-aged man should prompt the consideration of NS as a possible differential diagnosis. While steroid responsive, the majority of NS-CE/CM patients are left with residual neurodeficits with quick relapses when steroids are tapered, making the case for early institution of immunosuppressive therapies.
\end{abstract}

Categories: Neurology, Pathology, Rheumatology

Keywords: neurosarcoidosis, cauda equina, immunosuppressive therapy

\section{Introduction}

Sarcoidosis is an autoimmune multi-organ inflammatory disorder histologically characterized by diffuse infiltration of non-caseating granulomas, with an estimated incidence of 7.6-8.4/100, 000 (2010-2013) in the United States [1]. More than $90 \%$ cases present in the third or fourth decade of life with pulmonary involvement, skin or ocular disease, or a combination of these [1]. Nearly $25 \%$ patients have involvement of the central nervous system (CNS), but only around $10 \%$ actually become symptomatic. Spinal cord involvement is reported to occur in 1-18\% of all neurosarcoidosis (NS) cases [1]. Involvement of the cauda equina and/or the conus medullaris (CE/CM) is rare, with only a handful of cases reported worldwide. There are no current guidelines on the management of patients with CE/CM syndrome secondary to NS, with the current standard of care involving a variable taper of corticosteroids with or without long-term immunosuppression (IS) [2]. We present the case of a young man with NS -related CE/CM syndrome (NS$\mathrm{CE} / \mathrm{CM}$ ) who was treated with steroids with recurrent relapses until IS was initiated. We searched PubMed from database inception to April 2019 with the search terms "[cauda equina] OR [conus medullaris] AND

Received 06/22/2020 Review began 08/05/2020 Review ended 08/20/2020 Published 08/27/2020

\section{○ Copyright 2020}

Topiwala et al. This is an open access article distributed under the terms of the Creative Commons Attribution License CC-BY 4.0., which permits unrestricted use, distribution, and reproduction in any medium, provided the original author and source are credited. [sarcoidosis] or [neurosarcoidosis]" to review the current literature on the management and prognosis of NSCE/CM.

\section{Case Presentation}

A 49-year-old-Hispanic man with a 12-month history of Bell's palsy and 6-month history of hypogonadotropic-hypogonadism presented to the emergency room with four weeks of sudden-onset, progressive bilateral leg parasthesias and weakness. Neurological exam showed bilaterally asymmetric leg weakness (distal > proximal) with loss of deep tendon reflexes in both legs, except for the right patellar reflex; bilateral stocking neuropathy for large and small fiber modalities with a positive Romberg's sign; and a wide-based, high-steppage gait. The admission Expanded Disability Status Scale (EDSS) score was 7. Hypogonadism was diagnosed following a six-month history of unintentional weight loss of 40 pounds, dry cough, and low-grade fevers. Magnetic resonance imaging (MRI) brain with a pituitary-protocol completed six weeks prior to admission was normal. A complete blood count along with liver and kidney function tests on admission were unremarkable. Cerebrospinal fluid (CSF) analysis revealed that nucleated cells were 92/cubic (cu) mm (98\% were lymphocytes), red blood cells were $9 / \mathrm{cu} \mathrm{mm}$, total protein count was $131 \mathrm{mg} / \mathrm{dL}$, and glucose was $45 \mathrm{mg} / \mathrm{dL}$. CSF gram stain did not show any neutrophils or bacteria, and cultures did not demonstrate any growth. Similarly, Ziehl-Neelsen staining did not reveal any acid-fast bacilli, and mycobacterial cultures remained negative. CSF tests for syphilis, herpes simplex virus I/II, cytomegalovirus, and enterovirus through polymerase chain reaction were negative. West Nile virus immunoglobulin (Ig) $\mathrm{M} / \mathrm{IgG}$ antibodies and CSF cryptococcal antigen screen were negative as well. CSF cytology and flow cytometry were unremarkable. CSF oligoclonal bands were concordant with serum bands. CSF and serum angiotensin-converting enzyme (ACE) levels were within the normal range. CSF and serum paraneoplastic panels were unrevealing. Electromyography (EMG) and nerve conduction velocity (NCV) studies showed a subacute sensory-motor polyneuropathy with axonal and demyelinating features. MRI of the lumbar spine showed diffuse enhancement of the CE nerve roots (Figures 1A, 1B). MRI brain with pituitary protocol showed new diffuse thickening of the pituitary stalk ( $4 \mathrm{~mm}$ from $1 \mathrm{~mm}$ six weeks before admission), with mild enhancement (Figures $1 C, 1 D$ ). Computed tomography $(\mathrm{CT})$ scan of the chest, abdomen, and pelvis 


\section{Cureus}

showed enlargement of bilateral mediastinal lymph nodes (Figure 2), which were endoscopically biopsied, revealing non-caseating granulomas (Figures $1 E, 1 F$ ). He was diagnosed with probable NS according to the 2018 consensus criteria by Stern et al. [2] and started on a steroid taper $(0.5 \mathrm{mg} / \mathrm{kg}$ for four weeks) with significant improvement. He was able to ambulate without support on discharge at one week, with a sixweek EDSS score of 1. Repeat EMG six weeks later showed improvement. However, he became steroid dependent with quick recurrence of symptoms upon discontinuation of steroids. He was subsequently started on methotrexate $20 \mathrm{mg}$ per week, with dramatic improvement in strength. At 24-month follow-up, the patient reported stable symptoms, with no subsequent relapses.

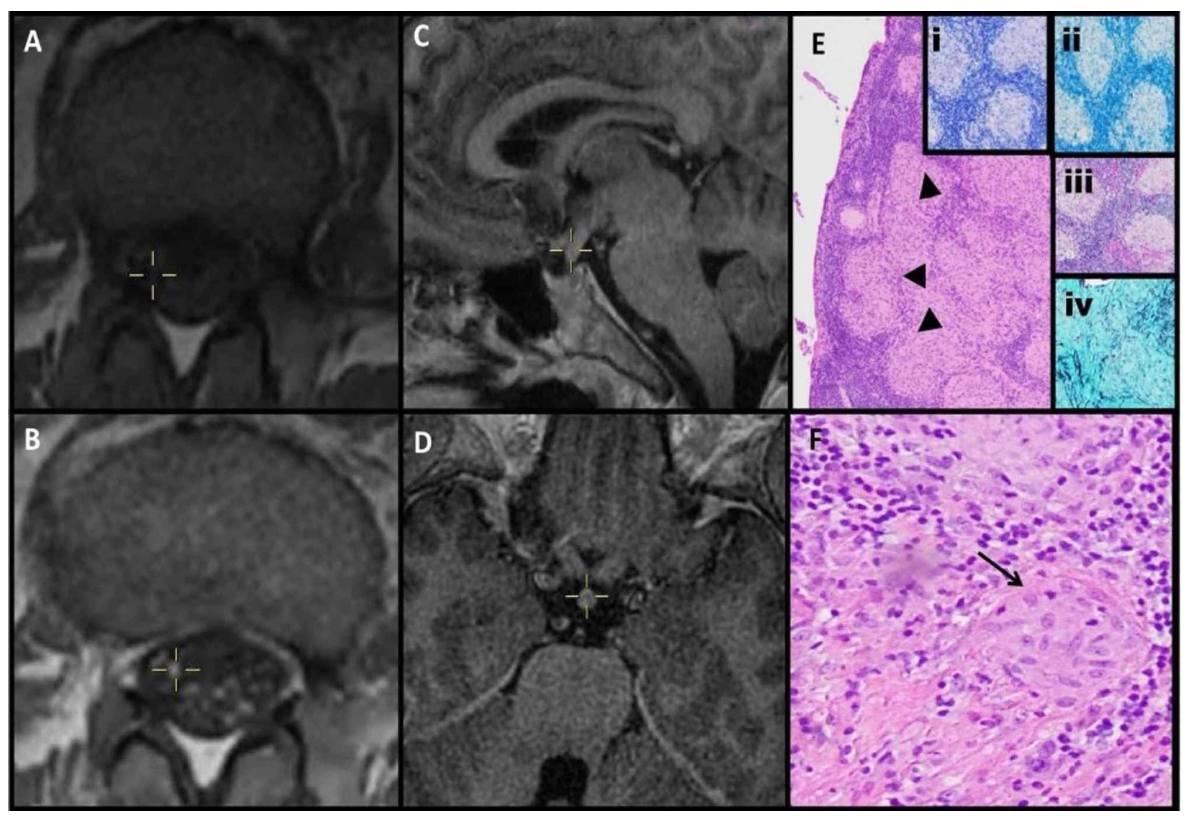

FIGURE 1: MRI of the lumbar spine showing axial sections through the cauda equina.

(A) Pre- and (B) post-contrast images show diffuse nodular enhancement. MRI brain volume imaging (BRAVO) sagittal (C) and axial (D) sections show diffuse enlargement with mild enhancement of the pituitary stalk. (E) Hematoxylin and eosin stain of hilar lymph nodes shows diffuse infiltration of non-caseating granulomas (arrowhead) of the epithelioid cells and multinucleated giant cells (arrow). Special staining with Ziehl-Neelsen (i) and Fite (ii) for mycobacteria, and periodic acid-Schiff (iii) and Gömöri methenamine (iv) for fungal elements was unremarkable.

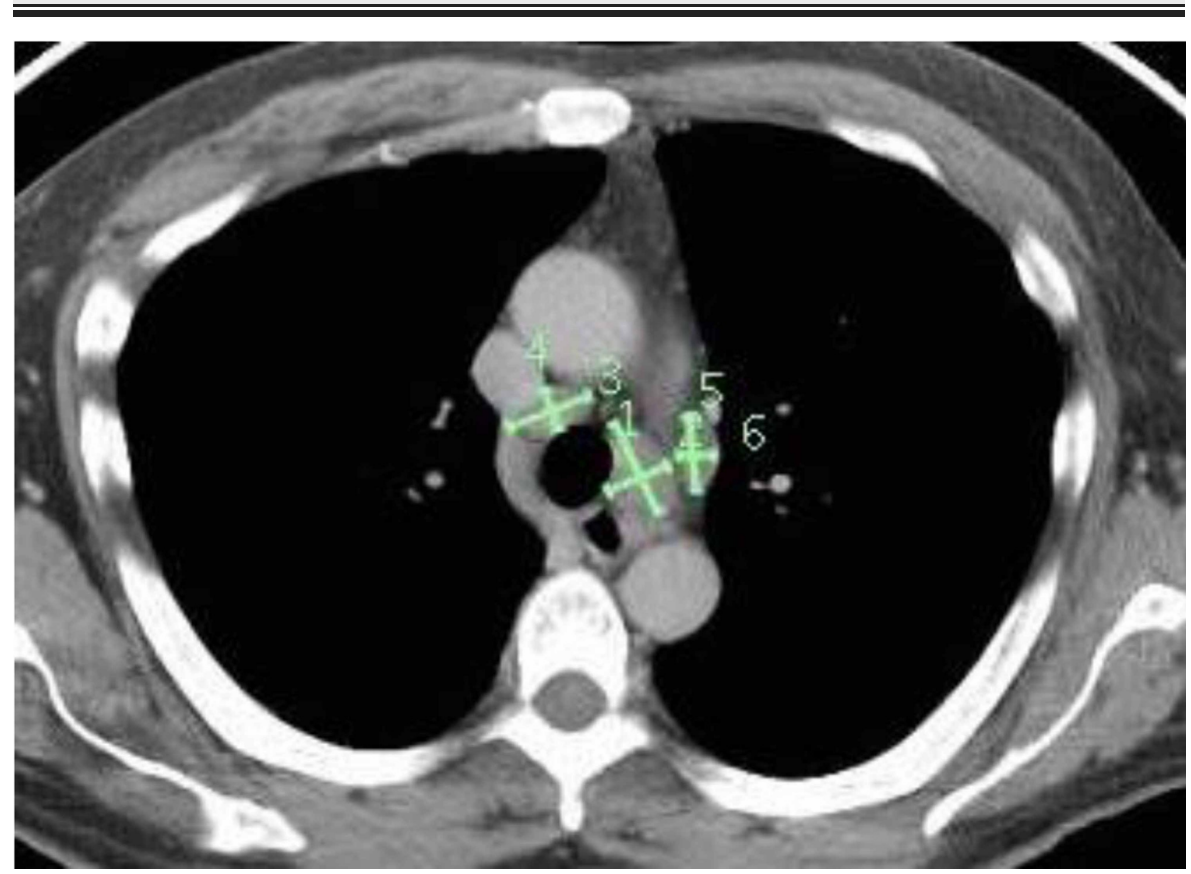

FIGURE 2: CT of the chest showing extensive mediastinal 
lymphadenopathy.

\section{Discussion}

Sarcoidosis is an autoimmune multi-system disorder characterized by the presence of non-caseating epithelioid granulomas on histopathology, with an estimated prevalence of 1 to 40 per 100,000 worldwide [1]. Involvement of the nervous system (central and/or peripheral) was first described in 1909 in three men with uveitis, parotid enlargement, fever, and facial palsy [2]. Although around 25\% of sarcoidosis patients have involvement of the CNS, only around $10 \%$ actually become symptomatic, with $50 \%$ to $70 \%$ of those presenting with only neurological symptoms [1]. The remainder will become symptomatic within two years of diagnosis [1]. Spinal cord involvement is reported to occur in 1-18\% of all NS cases [2]. In a meta-analysis of 1,088 NS patients, only $31 \%$ had evidence of systemic disease at presentation, but this increased to $84 \%$ later in the disease course [3]. Spinal cord involvement is reported to occur in $0.43 \%$ to $1 \%$ of all NS cases; however, the aforementioned meta-analysis found spinal cord disease in upto $18 \%$ NS cases. Such lesions could be enhancing and longitudinally extensive, and may even have a relapsing-remitting course [3]. This results in a wide differential diagnosis; however, an enhancing ( 96\%) intramedullary lesion ( 81\%) with leptomeningeal enhancement ( 48\%) spanning more than three spinal levels ( $77 \%)$ makes NS more likely [2]. It is suggested that parenchymal lesions result from the spread of leptomeningeal inflammation through the Virchow-Robin spaces, which are particularly large at the base of the brain, accounting for their preferential involvement. The lesions evolve in four phases: linear leptomeningeal enhancement, cord expansion, focal > multiple intra-medullary disease with diminishing enhancement, and eventually atrophy [3]. Additionally, extramedullary-intradural lesions (arachnoiditis, nerve root nodules, and root clumping) and extramedullary-extradural lesions (vertebral-body granulomas and discitis) have also been described.

The most common presenting features of NS are cranial neuropathies in 55\% (VII [24\%] > II [21\%] > V [12\%] > VIII [11\%]), headache in $32 \%$, sensory symptoms in $29 \%$, and motor weakness in $19 \%$ (hemiparesis [9\%], paraparesis [11\%]) [3]. Neuroendocrine dysfunction (pituitary-hypothalamic inflammation), meningeal disease (acute aseptic meningitis, leptomeningeal-pachymeningeal mass lesions), brain parenchymal lesions (mass effect, encephalitis, or seizures), acute-subacute hydrocephalus (communicating or noncommunicating) are common presentations [3]. However, CM/CE involvement is extremely rare, with a handful of case series to date.

A diagnosis of NS is typically established via a neural/non-neural biopsy demonstrating the pathologic hallmark of non-caseating epithelioid granulomas in the absence of an infection (tuberculosis, invasive fungal disease), inflammation (inflammatory bowel disease), malignancy (lymphoma), and foreign body/elements (silica) [1]. Consensus diagnostic criteria for NS were only recently published in June 2018 by the Neurosarcoidosis Consortium Consensus Group (10 neurologists and 4 pulmonologists) and endorsed by the World Association of Sarcoidosis and Other Granulomatous Disorders [2]. They require a presentation consistent with NS, both clinically as well as on investigational studies (MRI, CSF, and/or EMG/NCS), and a rigorous exclusion of other differential diagnoses. Thereafter, based on histopathological evidence, they were classified as possible (no pathological confirmation), probable (positive non-neural pathology), and definite (positive neural pathology [type A] or neural plus non-neural pathology [type B]) [2]. Probable and definite were considered to have NS. Thus, a biopsy is always required except in patients presenting with Löfgren's syndrome (arthritis, erythema nodosum, and bilateral hilar adenopathy, which are present in 9$34 \%$ of patients) [1]. Multiple CSF abnormalities have been described (lymphomonocytic pleocytosis [ 57\%], elevated protein [ 60\%], low glucose, elevated IgG index, and oligoclonal bands [ 20\%]) and are most likely in patients with leptomeningeal enhancement [1]. However, no CSF finding is considered pathognomonic of NS, with the CSF being completely normal in upto $30 \%$ cases [1]. Biomarkers (including ACE, lysozyme, Creactive protein, and soluble interleukin-2 receptor assays) in either CSF or serum have not been found to be sensitive nor specific and are not useful in making a diagnosis of NS [3]. Similar to our patient, chest imaging will be abnormal in upto 90\% patients [1]. Such imaging may include a chest X-ray, high-resolution chest CT scan or gallium citrate [Ga67] scintigraphy, or, preferably, fluorodeoxyglucose positron emission tomography (PET) scan with the aim of identifying a possible biopsy site [3].

\section{Literature review}

Most patients with systemic sarcoidosis do not require therapy [1]. However, currently, NS is primarily treated with systemic corticosteroids, and if it fails, an immunosuppressant is added [4]. The aggressiveness of initial treatment in NS has long been debated; however, in the absence of randomized controlled trials, open-labeled studies are the best available evidence. Scott et al. treated 43 patients with probable or definite NS and followed them for a mean $( \pm$ SD) of 44.1 months $( \pm 43.6)$ and found that of those treated with an IS (since or within six months of diagnosis) had higher rates of clinical improvement (69\% vs. 35\%) [4]. Joubert et al. followed 234 NS patients (one of the largest NS cohort studies available) for $>60$ months (in $68.4 \%$ cases) and found a $28 \%$ relapse-free survival rate with the use of an IS [4]. The 10-year survival rate was $89 \%$, with older age (HR: 1.64), peripheral nervous system disease (HR: 6.75), and higher baseline EDSS score (HR per point: 1.21) being associated with higher mortality. Despite good survival rates, the 10-year relapse rate was high (86.2\%) with encephalic disease [4]. Spinal NS is considered to be relatively refractory to corticosteroids, with one 29-patient series reporting an IS in 83\% cases [4]. Optimal management of NS when presenting as $\mathrm{CE} / \mathrm{CM}$ syndromes remains poorly studied, with variable response to corticosteroids. Contradictory to prior observations, in the study by Joubert et al., myelopathy was not associated with 


\section{Cureus}

poorer outcomes. Monoclonal antibodies such as infliximab have also been used in patients with chronic steroid-resistant pulmonary and NS with significant clinical and radiographic improvement [3]. A review of NS-CE/CM cases published to date (Table 1$)$ revealed that $68 \%(n=17)$ were men with a median age of 40.94 years (SE: 13.78). Of the patients, $35 \%(n=7 / 20)$ and $16 \%(n=3 / 19)$ had a history of prior systemic disease and neurological symptoms, respectively. The median duration of symptoms prior to presentation was 18 months (SE: 7.84). Biopsy was performed on neural tissue in $50 \%(n=11 / 22)$, non-neural tissue in $36 \%$ $(n=8 / 22)$, and both in $14 \%(n=3 / 22)$. Of the 22 patients, $68 \%(n=152)$ were treated with steroids only, with $23 \%(n=5)$ receiving an IS medication. The overall recovery was reported as moderate or poor in $87 \%$ ( $n=20 / 23)$, while only $13 \%(n=3 / 23)$ were asymptomatic at the last follow-up. Further research in the field is needed with multi-center randomized controlled trials assessing the role of simultaneous initiation of steroids along with long-term immunosuppressive agents in patients with NS-CE/CM syndrome.

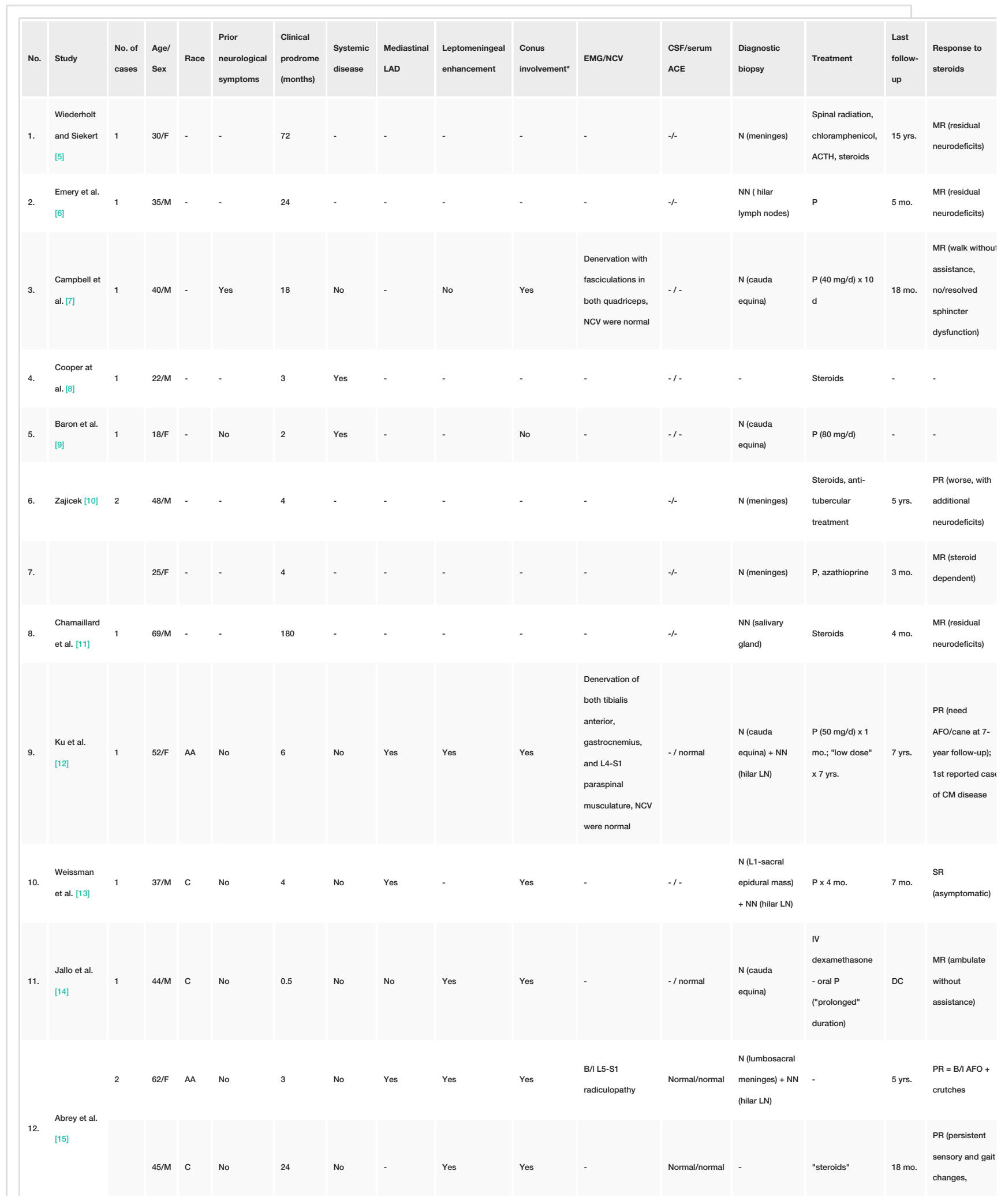




\section{Cureus}

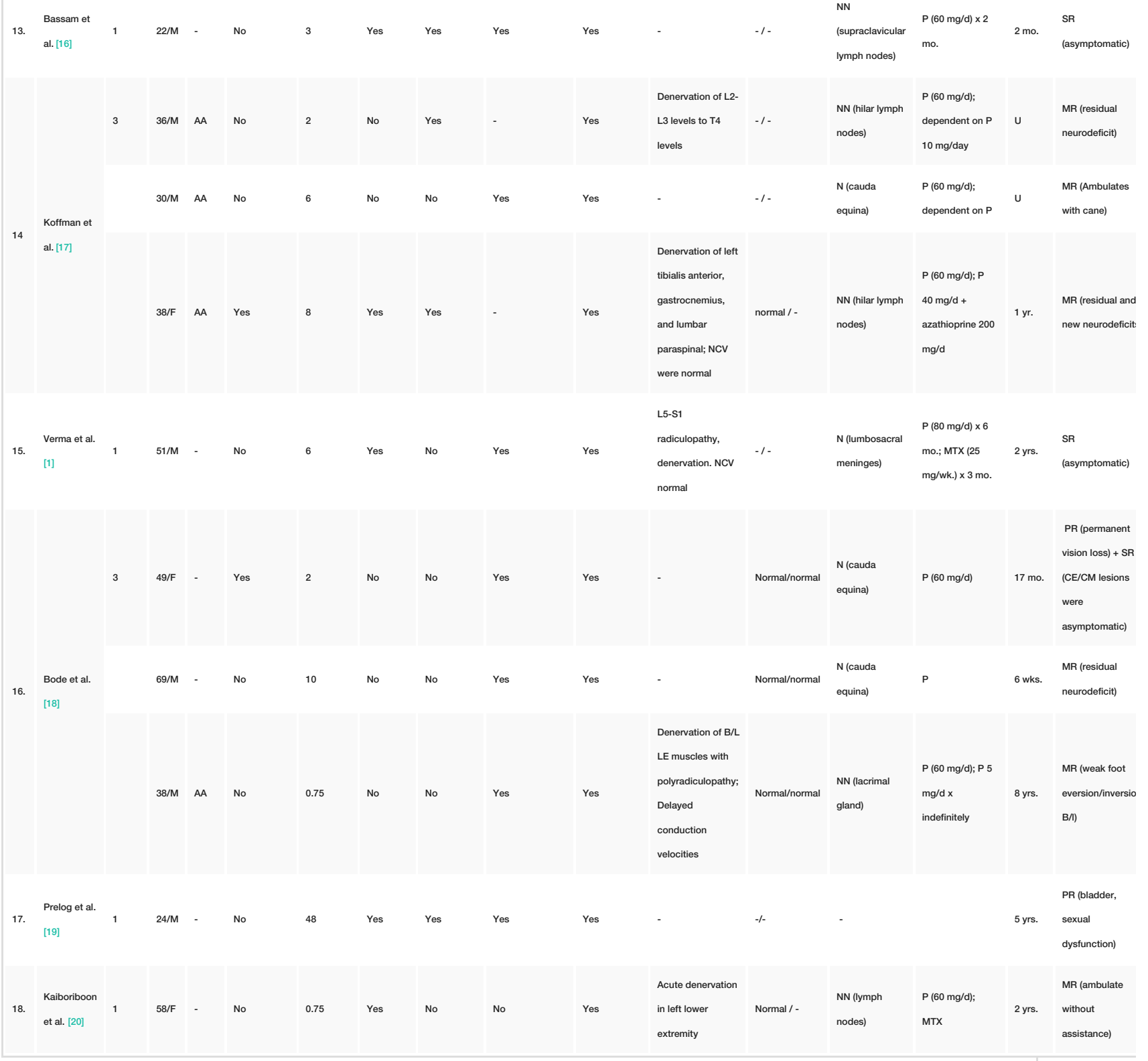

\section{TABLE 1: Review of published case reports of NS presenting with CE/CM syndromes}

AA, African-American; ACE, angiotensin-converting enzyme; ACTH, adrenocorticotrophic hormone; AFO, ankle-foot orthotics; C, Caucasian; CE, cauda equina; CM, conus medullaris; CSF, cerebrospinal fluid; D, day; EMG/NCV, electromyography and nerve-conduction-velocity; SR, significant response; MR, moderate response; LAD, lymphadenopathy; PR, poor response; P, prednisone; MTX, methotrexate; N, neural; NN, non-neural; NS, neurosarcoidosis; -, unavailable; B/l, bilateral

Note: conus involvement was either radiographic or defined as loss of ankle reflex along with an asymmetric loss of knee reflex, bowel or bladder dysfunction, erectile dysfunction, and saddle anesthesia.

\section{Conclusions}

Involvement of the spinal cord in NS is rare. The current body of evidence on the management and prognosis of patients with NS-CE/CM syndrome is based on short case series and suggests that while the disease is steroid responsive, the vast majority of these patients will be left with residual neurological deficits with quick relapses when steroids are tapered. Prospective studies evaluating the role of IS at initial presentation of spinal cord disease in patients with NS are urgently required to minimize the morbidity caused by this disorder.

\section{Additional Information}

Disclosures 
Human subjects: Consent was obtained by all participants in this study. University of Connecticut School of Medicine and Hartford Hospital issued approval NA. The Institutional Review Boards noted above exempt all case-reports and case-series containing less than equal to three patients from requiring an IRB approval. . Conflicts of interest: In compliance with the ICMJE uniform disclosure form, all authors declare the following: Payment/services info: All authors have declared that no financial support was received from any organization for the submitted work. Financial relationships: All authors have declared that they have no financial relationships at present or within the previous three years with any organizations that might have an interest in the submitted work. Other relationships: All authors have declared that there are no other relationships or activities that could appear to have influenced the submitted work.

\section{References}

1. Verma KK, Forman AD, Fuller GN, Dimachkie MM, Vriesendorp FJ: Cauda equina syndrome as the isolated presentation of sarcoidosis. J Neurol. 2000, 247:573-574. 10.1007/s004150070163

2. Stern BJ, Royal W, Gelfand JM, et al.: Definition and consensus diagnostic criteria for neurosarcoidosis from the Neurosarcoidosis Consortium Consensus Group. JAMA Neurol. 2018, 75:1546-1553. 10.1001/jamaneurol.2018.2295

3. Fritz D, vandeBeek D, Brouwer MC: Clinical features, treatment and outcome in neurosarcoidosis: systematic review and meta-analysis. BMC Neurol. 2016, 16:220. 10.1186/s12883-016-0741-x

4. Joubert B, Chapelon-Abric C, Biard L, et al.: Association of prognostic factors and immunosuppressive treatment with long-term outcomes in neurosarcoidosis. JAMA Neurol. 2017, 74:1336-1344. 10.1001/jamaneurol.2017.2492

5. Wiederholt WC, Siekert RG: Neurological manifestations of sarcoidosis. Neurology. 1965, 15:1147-1154. 10.1212/wnl.15.12.1147

6. Emery JP, Lasseree PP, Dryll A: Les manifestations neurologiques périphériques de la sarcoïdose [Peripheral neurologic manifestations of sarcoidosis]. Sem Hop. 1972, 48:3039-3043.

7. Campbell JN, Black P, Ostrow PT: Sarcoid of cauda equina. J Neurosurg. 1977, 47:109-112. 10.3171/jns.1977.47.1.0109

8. Cooper SD, Brady MB, Williams JP, Pilgreen KL, Harp DL, Weissmann JR: Neurosarcoidosis: evaluation using computed tomography and magnetic resonance imaging. J Comput Tomogr. 1988, 12:96-99. 10.1016/0149936X(88)90060-4

9. Baron B, Goldberg AL, Rothfus WE, Sherman RL: CT features of sarcoid infiltration of a lumbosacral nerve root. J Comput Assist Tomogr. 1989, 13:364-365. 10.1097/00004728-198903000-00041

10. Zajicek J: Sarcoidosis of the cauda equina: a report of three cases . J Neurol. 1990, 237:424-426. $10.1007 /$ bf00314734

11. Chamaillard S, Dubas F, Penission-Besnier I: Syndrome de la queue de cheval d'origine sarcoïdiosique. A propos d'un cas [Cauda equina syndrome in sarcoidosis. Report of a case]. Rev Rhum Mal Osteoartic. 1990, 57:901-901.

12. Ku A, Lachmann E, Tunkel R, Nagler W: Neurosarcoidosis of the conus medullaris and cauda equina presenting as paraparesis: case report and literature review. Paraplegia. 1996, 34:116-120. 10.1038/sc.1996.21

13. Weissman MN, Lange R, Kelley C, Belgea K, Abel L: Intraspinal epidural sarcoidosis: case report . Neurosurgery. 1996, 39:179-181. 10.1097/00006123-199607000-00041

14. Jallo GI, Zagzag D, Lee M, Deletis V, Moroto N, Epstein FJ: Intraspinal sarcoidosis: diagnosis and management. Surg Neurol. 1997, 48:514-521. 10.1016/S0090-3019(96)00440-5

15. Abrey LE, Rosenblum MK, DeAngelis LM: Sarcoidosis of the cauda equina mimicking leptomeningeal malignancy. J Neurooncol. 1998, 39:261-265. 10.1023/a:1005958205591

16. Bassam BA, Pilgreen KL, Mendizabal JE: Leg weakness in neurosarcoidosis. South Med J. 1998, 91:870-872. 10.1097/00007611-199809000-00015

17. Koffman B, Junck L, Elias SB, Feit HW, Levine SR: Polyradiculopathy in sarcoidosis. Muscle Nerve. 1999, 22:608-613. 10.1002/(SICI)1097-4598(199905)22:5<608::AID-MUS9>3.0.CO;2-L

18. Bode MK, Tikkakoski T, Tuisku S, Kronqvist E, Tuominen H: Isolated neurosarcoidosis - MR findings and pathologic correlation. Acta Radiologica. 2001, 42:563-567.

19. Prelog K, Blome S and Dennis C: Neurosarcoidosis of the conus medullaris and cauda equina . Australasian Radiol. 2003, 47:295-297. 10.1046/j.1440-1673.2003.01180.x

20. Kaiboriboon K, Olsen TJ, Hayat GR: Cauda equina and conus medullaris syndrome in sarcoidosis. case report and literature review. Neurologist. 2005, 11: 179-183. 10.1097/01.nrl.0000159983.19068.21 